\title{
Light-Meson Spectroscopy in Strong Magentic Field
}

\author{
Maxim Andreichikov* \\ Institute for Theoretical and Experimental Physics \\ E-mail: andreichicovemail.ru
}

\section{Boris Kerbikov}

Institute for Theoretical and Experimental Physics

Lebedev Physicsal Institute

Moscow Institute for Physics and Technology Affiliation

E-mail: boriskeitep.ru

\section{Yuri Simonov}

Institute for Theoretical and Experimental Physics

E-mail: simonoveitep.ru

\begin{abstract}
The spectra of charged and neutral $\rho$ and $\pi$ mesons in the framework of the vacuum correlator method obtained from the relativistic Hamiltonian in external homogeneous magnetic field (MF). The spectra of all 12 spin-isospin s-wave states generated by $q \bar{q}$ light mesons with different spin projections are calculated analytically as a function of the field strength. Three types of the asymptotic behaviour in strong field limit are found: two of them grow with MF and the last one tend to be a constant (zero mode). The mass of the zero mode becomes small in MF whcih can be the source of the meson collapse. The potential collapse has two different sources - colormagnetic hyperfine interaction and one-gluon exchange and it doesn't occur for the MF $e B<2 \mathrm{GeV}^{2}$. The results are in agreement with recent lattice calculations.
\end{abstract}

XVII International Conference on Hadron Spectroscopy and Structure

25-29 September, 2017

University of Salamanca, Salamanca, Spain

${ }^{*}$ Speaker. 


\section{Introduction}

High intense magnetic fields up to $e B \sim \Lambda_{Q C D}^{2} \sim 10^{19} G$ are generated during the early stages of heavy ion collisions at RHIC and LHC. The question is what happens with meson mass in strong MF $e B \sim m_{\pi}^{2}, m_{\rho}^{2}$. If quark dynamics inside the hadron is determined by confinement string with tension $\sigma \simeq(0.15-0.18) \mathrm{GeV}^{2}$, one can define critical MF as $B_{\sigma}=\sigma /|e| \simeq 10^{19} \mathrm{G}$. MFs stonger than $B_{\sigma}$ provide strong influence to the internal structure of hadron because Larmor radius of each quark $l_{B} \simeq 0.6 \mathrm{fm}$ is smaller than the typical hadron size. If one takes $\rho$ as an elementary particle it should collapse in MF when $m_{\rho}^{2}+e B\left(1-g_{\rho}\right)<0$. However, one should take to the account internal meson structure, when quark Larmor radius $l_{B}=\frac{1}{\sqrt{e B}}$ reaches the meson size $\sim \frac{1}{\sqrt{\sigma}}$. We calculated mass spectrum of the light mesons through the relativistic Hamiltonian was obtained with Vacuum Correlator Method (VCM) to see an interplay between the MF and confinement that prevents the collapse of the meson.

\section{Stochastic Vacuum Correlators Method}

Feynman-Fock-Schwinger representation for the meson Green's function in MF

$$
\begin{gathered}
G_{q_{1} \bar{q}_{2}}(x, y)=\int_{0}^{\infty} d s_{1} \int_{0}^{\infty} d s_{2}\left(D z^{(1)}\right)\left(D z^{(2)}\right)\left\langle\hat{T} W_{\sigma}(A)\right\rangle_{A} \times \exp \left(i e_{1} \int_{y}^{x} A_{\mu}^{(e)} d z_{\mu}^{(1)}-i e_{2} \int_{y}^{x} A_{\mu}^{(e)} d z_{\mu}^{(2)}\right) \times \\
\exp \left(e_{1} \int_{0}^{s_{1}} d \tau_{1}(\sigma \mathbf{B})-e_{2} \int_{0}^{s_{2}} d \tau_{2}(\sigma \mathbf{B})\right) \times \exp \left(-K_{1}-K_{2}\right)
\end{gathered}
$$

QCD vacuum is filled by the Euclidean stochastic gluonic field is characterized by gaussian correlator with the characteristic length $\lambda \sim 1 \mathrm{GeV}^{-1}$. Wilson loop $\left\langle\hat{T} W_{\sigma}(A)\right\rangle_{A}$ after the averaging over the vacuum background

$$
\left\langle W_{\sigma}(A)\right\rangle_{A}=\exp \left(-\int_{0}^{\tau_{E}} d t_{E}\left[\sigma\left|\mathbf{z}_{1}-\mathbf{z}_{2}\right|-\frac{4}{3} \frac{\alpha_{s}}{\left|\mathbf{z}_{1}-\mathbf{z}_{2}\right|}\right]\right)
$$

Proper times $s_{1}, s_{2}$ for each quark are considered as fluctuations around the Euclidean monotonous time $t_{E}$ in the absence of pair production proportional to $\sim \alpha^{4}$ (Z-graphs) in the leading order

$$
z_{4}(\tau)=t_{E}(\tau)+\Delta z_{4}(\tau), \omega_{i}=\frac{T}{2 s_{i}}, T=\left|x_{4}-y_{4}\right|
$$

Green's function in terms of relativistic Hamiltonian $H_{q \bar{q}}$ after the averaging over time fluctuations

$$
G_{q_{1} \bar{q}_{2}}(x, y)=\frac{T}{8 \pi} \int_{0}^{\infty} \frac{d \omega_{1}}{\omega_{1}^{3 / 2}} \frac{d \omega_{2}}{\omega_{2}^{3 / 2}}\langle\mathbf{x}| \operatorname{Tr}\left(\hat{T} e^{-H_{q_{1} \bar{q}_{2}} T}|\mathbf{y}\rangle\right.
$$

We are intersted in the ground state energy, $T \rightarrow \infty$ leads to the stationary point analysis for integrals over $\omega_{i}$

$$
\hat{H} \psi=M_{n} \psi, \frac{\partial M_{n}\left(\omega_{i}\right)}{\partial \omega_{i}}=0
$$


where $M_{n}$ is non-perturbative mass spectrum, $\omega_{i}$ - "dynamical" quark masses (constituent mass analog for heavy quarks). Total meson mass is a sum of non-perturbative part and first-order perturbative corrections

$$
M_{\text {total }}=M_{n}+\left\langle\psi\left|V_{O G E}\right| \psi\right\rangle+\left\langle\Psi\left|V_{S S}\right| \psi\right\rangle\left(\sigma_{1} \cdot \sigma_{2}\right)+\Delta M_{S E},
$$

where $\left\langle V_{O G E}\right\rangle$ - color Coulomb correction, spin-spin colormagnetic interaction $\left\langle V_{S S}\right\rangle$ and selfenergy $\Delta M_{S E}$ are originated from the 1-st order perturbative series of vacuum-averaged $\langle(\sigma B)(\sigma B)\rangle_{A}$ correlators.

\section{Relativistic Hamiltonian for Meson in MF}

Relativistic Hamiltonian for meson which included all non-perturbative dynamics has the form

$$
H_{q \bar{q}}=\sum_{i=1}^{2} \frac{\left(\mathbf{p}^{(i)}-e_{i} \mathbf{A}^{(i)}\right)^{2}+m_{i}^{2}+\omega_{i}^{2}+e_{i} \sigma_{i} \mathbf{B}}{2 \omega_{i}}+\sigma\left|\mathbf{z}^{(1)}-\mathbf{z}^{(2)}\right|
$$

Method of constituent separtation (CS) introduced in [1] provides an analytical solution of the spectral problem for neutral and charged mesons. In this picture quarks may be considered quasiindependent in $\perp \mathbf{B}$ plane for $e B \gg \sigma$. One can fix the c.m. position at the origin and to introduce an effective MF-dependent string tensions $\sigma_{i}\left(\sigma, e_{i} B\right)$ [1] to reproduce results were obtained for neutral mesons with Pseudomomentum procedure [2]

$$
\sigma\left|\mathbf{z}^{(1)}-\mathbf{z}^{(2)}\right| \rightarrow \sigma_{1}\left|\mathbf{z}^{(1)}-\mathbf{z}^{(c . m .)}\right|+\sigma_{2}\left|\mathbf{z}^{(2)}-\mathbf{z}^{(c . m .)}\right| .
$$

\section{Meson Trajectories Spltting}

Meson mass trajectories are splitted in MF due to the magnetic moment operator $\mathbf{B}\left(\frac{e_{1}}{2 \omega_{1}} \sigma_{1}+\frac{e_{2}}{2 \omega_{2}} \sigma_{2}\right)$ in (3.1) and hyperfine term from (2.6). $|++\rangle,|+-\rangle,|-+\rangle,|--\rangle$ basis in spin space is convenient for $e B \gg \sigma$ asymptotics analysis. Three types of behaviour are possible - zero field seeking (ZFS) asymptotic tends to be constant with MF increasing and two types of low field seeking (LFS1,LFS2) asymptotics grow with MF [1]:

- ZFS: $e_{1} \sigma_{1}^{z}>0, e_{2} \sigma_{2}^{z}>0: M_{Z F S}(e B \rightarrow \infty)=2 \sqrt{\sigma}$

- LFS1: $e_{1} \sigma_{1}^{z}>0, e_{2} \sigma_{2}^{z}>0: M_{L F S 1}(e B \rightarrow \infty)=\sqrt{e_{1} B}+\sqrt{2 \sigma}$

- LFS2: $e_{1} \sigma_{1}^{z}<0, e_{2} \sigma_{2}^{z}<0: M_{L F S 2}(e B \rightarrow \infty)=2 \sqrt{e_{1} B}+2 \sqrt{e_{2} B}$

There are 12 mass trajectories for $(\pi, \rho)$ mesons composed from $u, d$ quarks and antiquarks due to spin-isospin splitting (see Fig. 2,3,4).

\section{Perturbative Corrections and Collapse Prevention}

Color Coulomb interaction is a potential source of the collapse for ZFS states because of the unbounded fall of the $\left\langle V_{\text {oge }}\right\rangle$ matrix elemnt (dashed line) (see Fig.1) at $e B \sim 10 \mathrm{GeV}^{2}$. Virtual $q \bar{q}$-pairs embedded into MF screen the Coulomb potential (solid line) and prevent the collapse [3]. 
The second source of the collapse is due to the colormagnetic spin-spin interaction

$$
V_{S S}=\frac{8 \pi \alpha_{s}}{9 \omega_{1} \omega_{2}} \delta(\mathbf{r})\left(\sigma_{1} \cdot \sigma_{2}\right)
$$

In strong MF the ground state wave function acquires the form of an ellipsoid elongated in the direction of the MF with characteristic radii $r_{\perp} \simeq \sqrt{\frac{2}{e B}}, r_{z} \simeq \frac{1}{\sqrt{\sigma}}$. This means the magnetic "focusing" of the wave function $|\psi(0)|^{2} \sim e B$ at the origin and provides boundless decrease of $\left\langle V_{S S}\right\rangle$. The collapse is prevented due to the natural cutoff parameter in VCM for (5.1)

$$
\delta(\mathbf{r}) \rightarrow\left(\frac{1}{\lambda \sqrt{\pi}}\right)^{3} e^{-\frac{r^{2}}{\lambda^{2}}} ; \lambda \sim 1 \mathrm{GeV}^{-1},
$$

where $\lambda$ is the correlation length of the stochastic vacuum (gluelump length). We also note that in [6] a general theorem was proven according to which the eigenvalues of the relativistic Hamiltonian in MF are positive.

These two scenarios prevent collapse for all ZFS except the $\pi^{0}$ meson [1]. Chiral properties should be consided to explain an absence of the collapse in MF for the $\pi^{0}$.

\section{Pion Chiral Dynamics in MF}

Chiral properties of the $\pi$ meson were restored with Gell-Mann-Oaks-Renner relation

$$
m_{\pi}^{2} f_{\pi}^{2}=m_{q}|\langle\bar{u} u+\bar{d} d\rangle| ;\langle\bar{q} q\rangle=N_{c} M(0) \sum_{n=0}^{\infty}\left(\frac{\left.\psi_{n}^{(+-)}(0)\right|^{2}}{2 m_{n}^{(+-)}}+\frac{\left.\psi_{n}^{(-+)}(0)\right|^{2}}{2 m_{n}^{(-+)}}\right),
$$

where the chiral condensates $\langle\bar{q} q\rangle \sim e B$ and decay constants $f_{\pi}$ were calculated with VCM method and effective chiral lagrangian in terms of non-chiral meson spectrum [1]. One can see the resulting mass trajectories for chiral $\pi^{0}$ in Fig. 3 and for $\pi^{-}$in Fig.4.

\section{Resulting Meson Spectra in MF and Conclusions}

Below we present the results of our analytic calculations in comparison with the recent lattice results from $[1,4,5]$. The $\rho^{-}$meson mass evolution in MF is shown in Fig. 2. In Fig.3 results for $\pi^{0}$ and $\rho^{0}$ are exposed. Here we emphasize that $u \bar{u}$ and $d \bar{d}$ components give rise to their own trajectories with scale factor $\sqrt{2}$. In Fig. 4 we present mass evolution of chiral and non-chiral $\pi^{-}$ in comparison with lattice data.

In the presented work we have calculated the trajctories of $\pi$ and $\rho$ masses as functions of the external MF. An internal meson structure and pion chiral dynamics were taken into account. An expicit calculation shows an absence of the collapse for $0<e B<2 \mathrm{GeV}^{2}$. Two possible sources of the collapse were discussed. The analytic calculations of meson mass trajectories give results which are in agreement with recent lattice data $[4,5,7]$.

\section{Acknowledgement}

The authors are grateful to E.V.Luschevskaya and O.E.Solovjeva for discussions. The authors are supported by the Russian Science Foundation grant number 16-12-10414. 


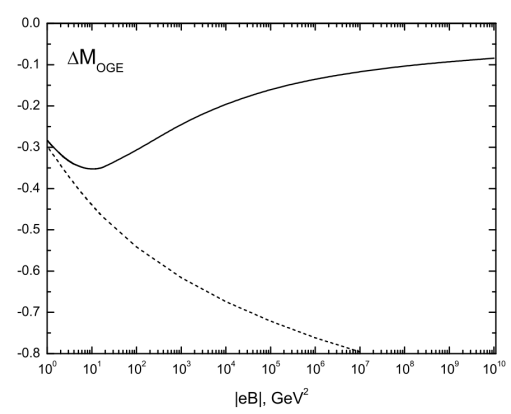

Figure 1: Color Coulomb potential with screening by the $q \bar{q}$ pairs in $\mathrm{MF}$ (solid line) and without screening (dashed line). The saturation of the $\left\langle V_{O G E}\right\rangle$ matrix element prevents the "hadron Coulomb catastrophe".

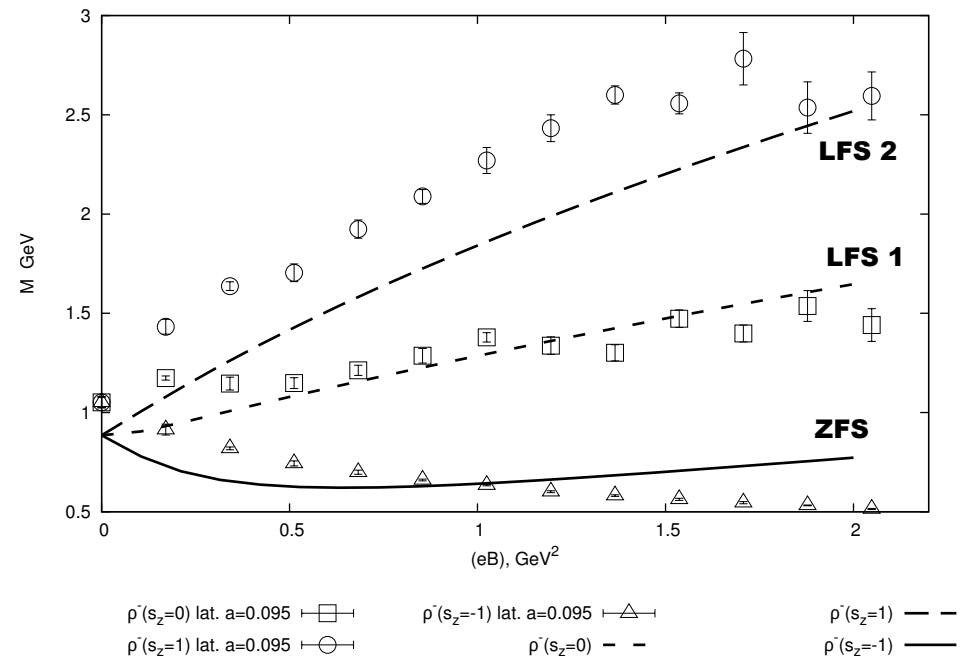

Figure 2: $\rho^{-}$meson trajectories in comparison with lattice data from [1]

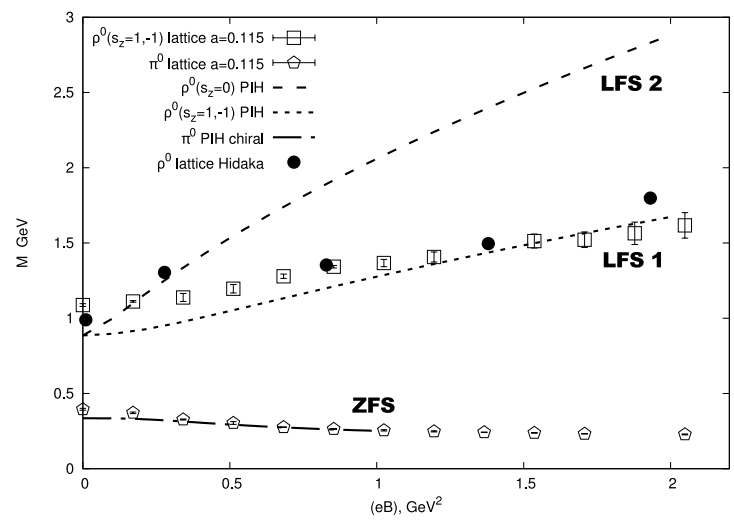

Figure 3: $\left(\pi^{0}, \rho^{0}\right)$ trajectories in MF for $u \bar{u}$ quark constituents. Another 4 trajectories for $d \bar{d}$ demonstrate the same behaviour up to the scale factor $\sqrt{2}$ is defined by the $q_{u} / q_{d}$ ratio. Lattice data is from $[1,5]$

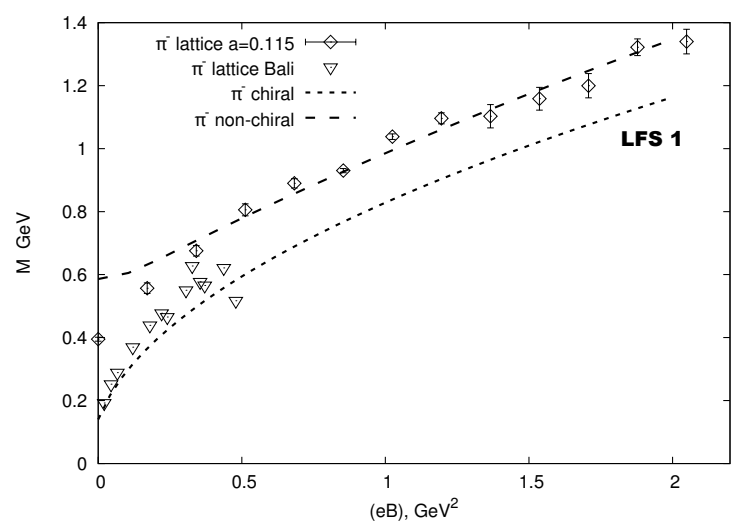

Figure 4: Chiral $\pi^{-}$meson (dotted line) loose its chiral properties with MF $e B>0.5 \mathrm{GeV}^{2}$ and becomes non-chiral (dashed line). Analytcal results are in good agreement with lattice data from $[1,4]$

\section{References}

[1] M.Andreichikov, B.Kerbikov, E.Luschevskaya, Yu.Simonov, O.Solovjeva, JHEP 1705, 007 (2017)

[2] M.Andreichikov, B.Kerbikov, V.Orlovsky, Yu.Simonov, Phys. Rev. D87, 094029 (2013)

[3] M.Andreichikov, B.Kerbikov, Yu.Simonov, Phys. Rev. Lett. 110, 162002 (2013)

[4] G.Bali, B.Brandt, G.Endrodi, B.Glasse, arXiv:1510.03899 (2015)

[5] Y. Hidaka, A.Yamamoto, Phys. Rev. D87, 094502 (2013)

[6] Yu.A.Simonov, Phys. Rev. D88, 053004 (2013).

[7] G.Bali, B.Brandt, G.Endrodi, B.Glasse, arXiv:1707.05600 (2017). 